Citation: Amouzad Mahdiraji, E., Sedghi Amiri, M., "Improving the Accuracy of the State Estimation Algorithm in the Power System Based on the Location of PMUs and Voltage Angle Relationships". Journal of Engineering Technology and Applied Sciences 5 (3) 2020 : 133-147.

\title{
IMPROVING THE ACCURACY OF THE STATE ESTIMATION ALGORITHM IN THE POWER SYSTEM BASED ON THE LOCATION OF PMUS AND VOLTAGE ANGLE RELATIONSHIPS
}

\author{
Ebadollah Amouzad Mahdiraji ${ }^{*}$ (D), Mojtaba Sedghi Amiri ${ }^{b}$ \\ Department of Engineering, Sari Branch, Islamic Azad University, Sari, Iran \\ ebad.amouzad@gmail.com (*corresponding author) \\ Neka Power Generation Management Company \\ amojtabasedgheamiri@yahoo.com
}

\begin{abstract}
In order to make better use of the power system, monitoring network mode variables is of particular importance, because these variables play an effective role in improving economic efficiency, improving network reliability and improving the ability to analyze the status of the system. For this purpose, state estimation algorithms have been used with the aim of accurately estimating state variables with finite measurements. Since today's measuring devices such as PMUs, in addition to measuring electrical quantities, are able to measure the voltage angle of the buses, this paper presents a method to make more accurate estimates. Obtained from all network variables. The proposed algorithm, while determining the number of measuring devices (PMU), determines their suitable location in such a way that using their information, the most accurate estimates can be obtained to obtain state variables and quantities. Electric provided. Increasing the accuracy of the calculations by using the derivative equations of the voltageangle equations of the buses coincides with the state estimation relations. Finally, the state estimation calculations were performed by the weighted least squares (WLS) method. The calculations were performed on the IEEE 14 bus network using MATLAB and MATPOWR software. The results show that the proposed method has been successful in increasing the accuracy of estimating state variables and reducing the number of PMUs and proper placement of PMUs.
\end{abstract}

Keywords: Phasor measurement unit, load estimation, state estimation algorithm, voltage angle, PMU location 


\section{Introduction}

Proper management of power systems requires access to complete system information, including accurate load measurement. The advantages of accessing accurate system information include improving economic efficiency, improving the reliability of power networks, its optimal control, improving the ability to analyze the system after a fault, and advanced protection based on measured quantities. With the development of power systems, monitoring and control of the system become more important for the proper functioning of power networks. Proper management of electrical energy will be possible when senior managers have a clear view of the energy demand process. Therefore, it is necessary to control a power system to evaluate the state variables (amplitude and voltage angle of the buses) of a network, but considering the economic constraints and the extent of power networks, the simultaneous measurement of almost all electrical variables it's impossible. Therefore, to obtain all the electrical variables, by measuring a number of them, other parameters are calculated using the state estimation method. State Estimation Provides the best estimate of system state vectors with measured information in the shortest possible time. Since the introduction of state estimation algorithms, various methods have been proposed to obtain the vector of state variables. To estimate the state of a network, it is necessary to make measurements such as measuring the active and reactive power injected into the bus and the power transmitted between the lines, and the voltage range of the bus. Today, using phasor measurement unit devices (PMUs), state estimation is done more accurately. These devices are synchronized with each other using the GPS system to be able to have the measured values simultaneously. PMUs can also extract the transmission current between the buses and the voltage angle of the buses and allow their relationships to be added to the $\mathrm{H}$ measurement matrix to improve the accuracy of estimating the state of the network variables. In connection with improving the accuracy of state estimation, designs and researches such as Kalman filter combination and least squares (LS) method error in power systems [1] to [6], using different statistical criteria to perform load estimation and method Various methods have been proposed to reduce the fault as well as optimize the number of measuring devices [7] and [8]. In [9] according to the past load information and real-time measurement in some limited points of the network, it is possible to properly estimate the network load, and to reduce the error, artificial intelligence method has been used. In many studies, the WLS statistical criterion has been used to estimate the network load [10] to [13]. Other measures taken to make the best use of the assigned capacity in the distribution network are network monitoring and monitoring, which is the most important tool for establishing a DMS system in distribution networks. In this regard, the need for load estimation in operation management is felt to a great extent [14] and [15]. The use of PMUs, or in other words, the use of new technology in the field of load estimation, has led to an increase in accuracy and high speed in estimating information [16] and [17]. Various methods have been proposed for locating measuring equipment. In some of these methods, an attempt has been made to reduce the number of measuring instruments available by observing the visibility of the system. In others, regardless of the existing measuring instruments and according to the system topology, a new placement has been done from the beginning [18]. In [19], by providing an incremental method that works based on the calculated variance of the bus voltage, a measurement device has been placed to make the system visible. In [20] the use of Cholesky analysis for optimal placement without duplication of measuring instruments is proposed. In [21], some measuring devices are first positioned so that the visibility of the network is ensured. Then, by reducing and relocating the equipment, an attempt has been made to reduce the cost of the entire system. In [22], first, the network is divided into smaller networks, and then in each section, the measuring equipment is placed separately. The use of special vector centrifuges and order centrifuges derived from the intersection matrix of a node with a branch 
is introduced in [23]. In [24], the sequential elimination procedure method is presented, which greatly reduces the number of cases in this method. Another method used to locate measuring instruments is the binary genetic algorithm, in which the objective function is the same as the cost of preparing and installing the measuring instruments [25] to [27].

In this paper, a new method for optimizing the estimation of state variables is presented. This method is based on the ability of PMUs to determine the voltage angle of the busses simultaneously. In this method, if it is installed in two buses adjacent to the PMU, the difference in voltage angle between the two buses will be in hand, so the derivative of the voltage-angle equations can be added to the $\mathrm{H}$ measurement matrix and finally increase the accuracy of state estimation calculations. In the following and in the second part, the theory of state estimation is presented. The third section discusses how to increase the accuracy of the estimate. Network simulation and its results are presented in the fourth section and finally in the fifth section the conclusion is presented.

\section{State estimation theory}

State estimation is the act of assigning a value to an unknown state system variable based on a finite measurement of many quantities in the system. Various comparison criteria can be used to estimate network parameters. In power systems, the state variables are a network $(\theta, \mathrm{V})$ and the proposed methods are based on the measurement theory of some electrical quantities such as I, V, and P. A common benchmark is to sum the squares to minimize the difference between the estimated and measured values. Weighted least squares (WLS) is one of the most common methods of estimating the state. The state estimation algorithm is often used to correct the fault of time-measuring devices in networks. The problem of estimating the state is to minimize a nonlinear objective function by considering a set of nonlinear unequal constraints, and minimizing the fault between the measured value and the actual value is rare. Assuming that we have a set of meters, we can obtain a relationship based on the measured value and the actual value of the desired parameters, which can be expressed as (1):

$Z=h(x)+e$

Where $Z$ is the vector of the measured values and can be real or virtual (initial guesses), $x$ is the vector of system state variables, $h$ is the vector of nonlinear functions that relate the measured values to the related state variables makes and $e$ is the fault model in each measuring device, which usually has a normal distribution and zero mean [1] and [7]. The vector of state variables is (2).

$x=\left(\left|V_{1}\right|,\left|V_{2}\right|, \ldots,\left|V_{N}\right|, \theta_{1}, \theta_{2}, \ldots, \theta_{N}\right)$

The goal in this method is to reduce the sum of squares of faults between the measured and actual values, which is done according to the weighting $(w)$ of the fault of each device. Therefore, the optimization objective function in the WLS method is as follows (3).

$\operatorname{minJ}(x)=$

$\sum_{i=1}^{m} w_{i}\left(z_{i}-h_{i}(x)\right)^{2}=[z-h(x)]^{T} w[z-h(x)]=[z-h(x)]^{T}[R]^{-1}[z-h(x)]$

Where $R$ is the diameter matrix of covariance of measurement faults and its elements are related to the amount of variance of measurement faults. By deriving from (3) and then by expanding Taylor and using the iteration method, state variables can be estimated using (4) [7]. 
$\left.\Delta X^{e s t}=[[H]]^{T}[R]^{-1}[H]\right]^{-1}[H]^{T}[R]^{-1}[Z-h(x)]$

Where the matrix $\mathrm{H}$ is expressed by (5):

$H=\left[\begin{array}{cc}\frac{\partial P_{\text {inj }}}{\partial \theta} & \frac{\partial P_{\text {inj }}}{\partial V} \\ \frac{\partial P_{\text {flow }}}{\partial \theta} & \frac{\partial P_{\text {flow }}}{\partial V} \\ \frac{\partial Q_{\text {inj }}}{\partial \theta} & \frac{\partial Q_{\text {inj }}}{\partial V} \\ \frac{\partial Q_{\text {flow }}}{\partial \theta} & \frac{\partial Q_{\text {flow }}}{\partial V} \\ \frac{\partial I_{\text {mag }}}{\partial \theta} & \frac{\partial I_{\text {mag }}}{\partial V} \\ \frac{\partial V_{\text {mag }}}{\partial \theta} & \frac{\partial V_{\text {mag }}}{\partial V}\end{array}\right]$

In it, the matrices $I, Q, P$, and $V$ represent the active power, reactive power, current, and voltage, respectively, and inj indicates the input to the bus, flow indicates the power transmitted between the two buses and mag represents the amplitude.

\section{The proposed algorithm}

In the proposed method, we want to locate and determine the minimum number of new measuring equipment (PMUs) and use the information measured by them, while improving the accuracy of the estimation method to achieve the best response for the committees. Therefore, the algorithm is expressed in two parts:

- Determining the number of equipment and their suitable location,

- Provide improvements to the state estimation method

\subsection{Location of measuring devices}

In the methods of estimating the location of measuring devices with two purposes: one is to determine the minimum number of measuring devices and select their appropriate location by considering the visibility conditions of the network and the other is to reduce the fault or In other words, minimizing the estimation fault. Therefore, the objective function is determined to meet the above two objectives.

In the proposed method, genetic algorithm is used in order to optimally position the devices. For this purpose, in the first step, with the information and specifications of the network, the load distribution is performed and the information obtained from the load distribution is used as a reference to calculate the objective function. In a genetic algorithm, each chromosome has a length equal to the number of locations where it can be measured. The genetic algorithm variable is based on the binary variable. In this optimization process, the binary variable indicates the presence or absence of meters in the desired location with the number one or zero. The number of chromosome units indicates the number of devices used, one of which is to minimize them. Therefore, the variable $\mathrm{Nz}_{\mathrm{z}}$ is assigned to it and is presented as a part of the objective function of the number of devices, during which the location of the device is also determined based on (6). 
In each iteration of the algorithm, when the location of the device is specified, a set of measurement values are calculated and according to (7) according to the studies on the subject of state estimation, its estimation fault is calculated. Is the reduction of this fault, so the estimation fault is calculated according to (7).

trance $\left(H^{T} R^{-1} H\right)=$ The objective function estimation fault

Where trace is a function that calculates the sum of the elements on the diameter of the matrix (which are weighted fault squares). Since the range of changes of these two parts of the objective function is not in the same range, these two functions are weighted and the main objective function of this part is presented as (8):

$\min \left(w_{1} \times \operatorname{trace}\left(H^{T} R^{-1} H\right)+w_{2} \times N_{2}\right)=$ objective function

Where $\mathrm{w}_{1}$ and $\mathrm{w}_{2}$ with constant change during the execution of the algorithm are obtained as trial and error equal to 1 and $17 \times 10^{7} / c$, respectively, where $c$ is the length of the chromosome.

According to the presented materials, the main steps of the location algorithm are as follows:

1) At the beginning of the positioning algorithm, load distribution is performed to receive the reference values of the amplitude and voltage phase.

2) An initial population is then generated for the genetic algorithm, which means that these initial populations are random combinations from the place where the meters are installed.

3) Having this population, the $\mathrm{H}$ matrix is formed and the corresponding $\mathrm{R}$ matrix is formed, and finally, the main objective function for each chromosome in each generation is calculated using the iteration algorithm presented in the next section.

4) According to the criteria of the genetic algorithm, the optimal chromosomes are separated and passed on to the next generation.

5) In the next step, the new generation will be updated and the operators will be applied and the next generation will be generated in the algorithm.

6) Returns to step 3 if needed in the final algorithm.

\subsection{Modification of $\mathbf{H}$ measurement matrix}

Based on the information obtained from PMUs, the positive component of voltage and currents at the time of measurement is accurately calculated on a microsecond scale and thus their phase angle is extracted. If two PMUs are placed on two adjacent buses, $\boldsymbol{\theta}_{\mathrm{ij}}$ can be extracted from relation $\mathrm{I}_{\mathrm{ij}}$ using (9) and (10).

$$
\begin{aligned}
& \cos \theta_{i j}=\frac{V_{i}^{2}+V_{J}^{2}-I_{i j}^{2} \times\left(R_{i j}^{2}+X_{i j}^{2}\right)}{2 \times V_{i} \times V_{j}} \\
& \theta_{i j}=\arccos \frac{V_{i}^{2}+V_{j}^{2}-I_{i j}^{2} \times\left(R_{i j}^{2}+X_{i j}^{2}\right)}{2 \times V_{i} \times V_{j}}
\end{aligned}
$$


In which $\mathrm{R}_{\mathrm{ij}}$ and $\mathrm{X}_{\mathrm{ij}}$ are the impedance values connected between the two buses $\mathrm{i}$ and $\mathrm{j}$, so the phase difference between the two buses connected to each other can be obtained and according to (10) can be Deriving from it added components to the components of the $\mathrm{H}$ matrix. In other words, the dimensions of the measurement matrix $\mathrm{H}$ can be increased, in which case the vectors $\partial \theta_{i j} / \partial \theta$ and $\partial \theta_{i j} / \partial V$ are added to the matrix and the matrix $\mathrm{H}$ in the form (11).

$H=\left[\begin{array}{cc}\frac{\partial P_{\text {inj }}}{\partial \theta} & \frac{\partial P_{\text {inj }}}{\partial V} \\ \frac{\partial P_{\text {flow }}}{\partial \theta} & \frac{\partial P_{\text {flow }}}{\partial V} \\ \frac{\partial Q_{\text {inj }}}{\partial \theta} & \frac{\partial Q_{\text {inj }}}{\partial V} \\ \frac{\partial Q_{\text {flow }}}{\partial \theta} & \frac{\partial Q_{\text {flow }}}{\partial V} \\ \frac{\partial I_{\text {mag }}}{\partial \theta} & \frac{\partial I_{\text {mag }}}{\partial V} \\ \frac{\partial V_{\text {mag }}}{\partial \theta} & \frac{\partial V_{\text {mag }}}{\partial V} \\ \frac{\partial \theta_{i j}}{\partial \theta} & \frac{\partial \theta_{\text {inj }}}{\partial V}\end{array}\right]$

At this stage, assuming that the number of meters and their location are known, the state is estimated. This section is presented as the iteration method algorithm. 


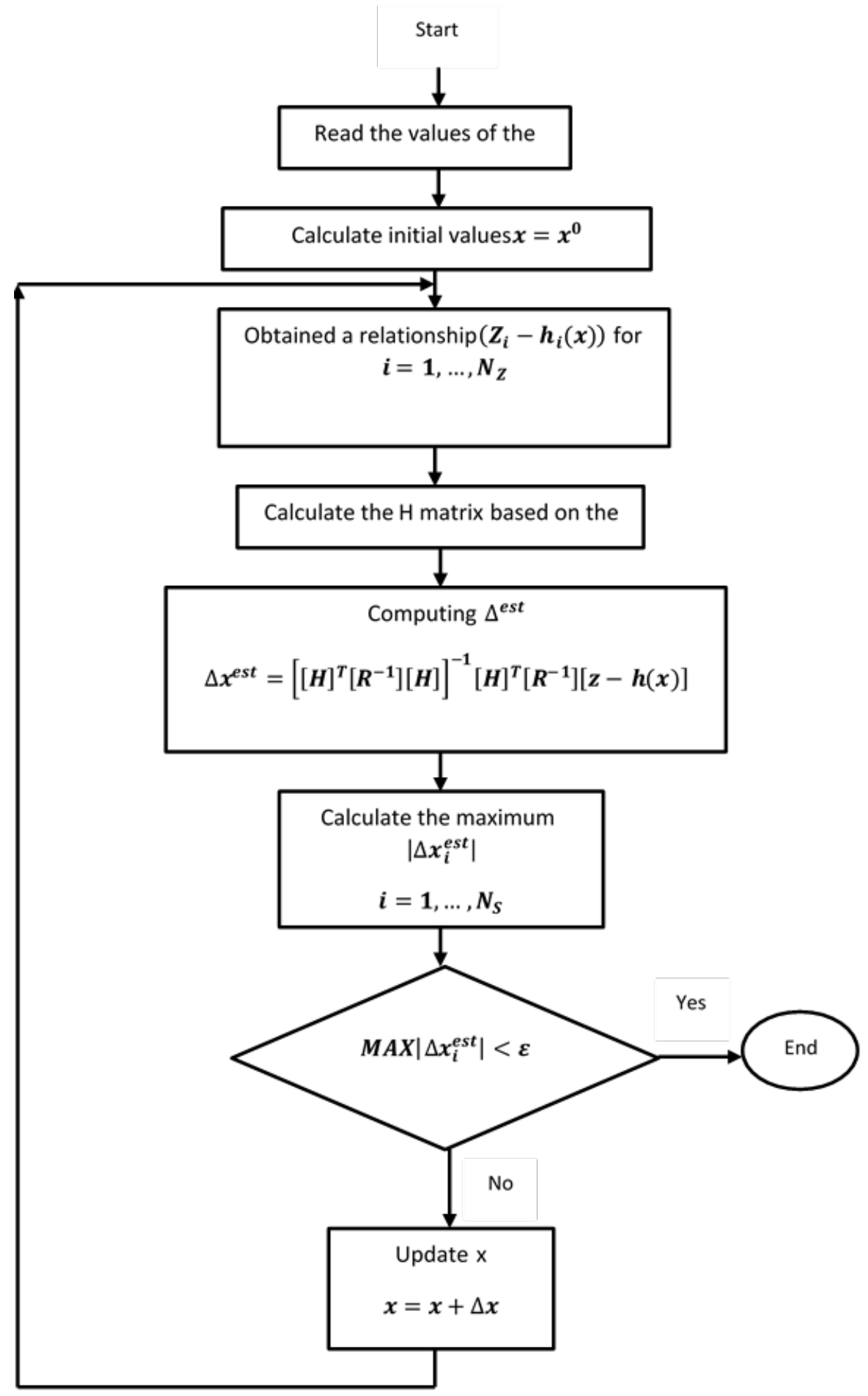

Figure 1. Iteration of algorithm process 


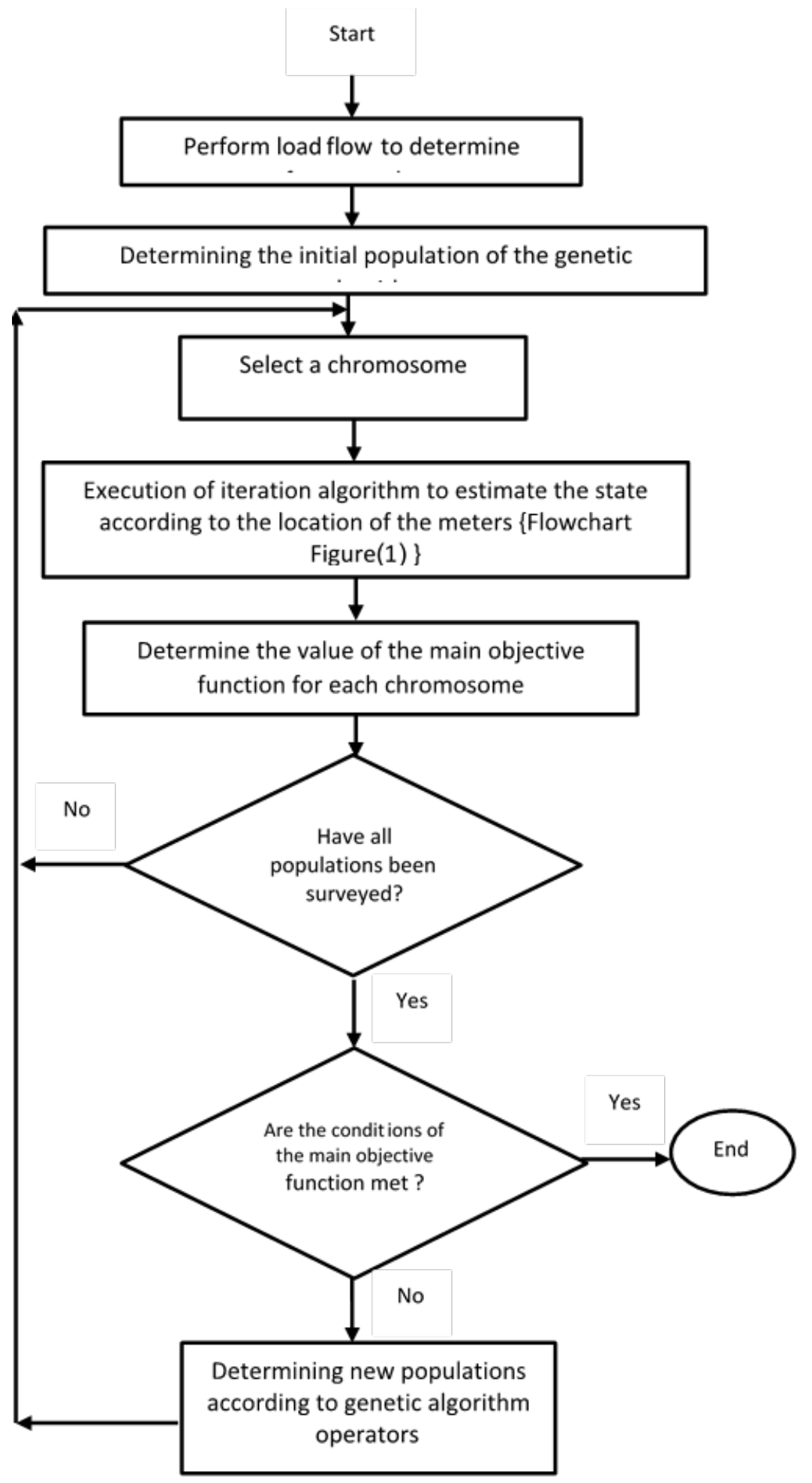

Figure 2. The final process of locating PMUs and state estimating of parameters 


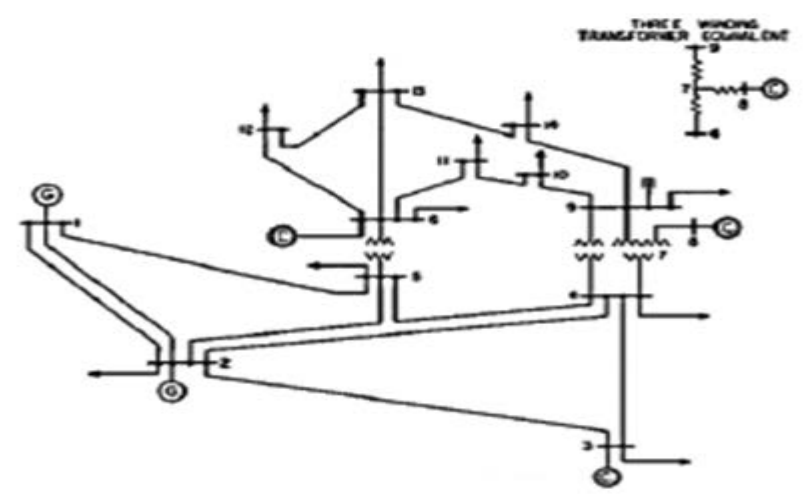

Figure 3. IEEE 14-bus network

According to Figure 1, the following steps are as follows:

1. At the beginning of the algorithm, the values of the measuring devices (specified in the location algorithm) are read.

2. By load loading, the $\mathrm{X}^{\mathrm{k}}$ vector becomes the starting point for initialization.

3. The relation $z_{i}-h_{i}\left(x^{k}\right)$ is calculated.

4. The matrix $H\left(x^{k}\right)$ is calculated from (11).

5. Using (4), $\Delta \mathrm{x}^{\mathrm{k}}$ is calculated and then max $\max \left|\Delta x_{i}^{\text {est }}\right|$ In other words, the maximum values of the estimated variables are separated.

6. The condition max $\max \left|\Delta x_{i}^{\text {est }}\right|<\varepsilon$ is evaluated.

7. If the condition is not met, the values of $x^{k+1}=x^{k}+\Delta x^{k}$ and $k=k+1$ are updated. Then it will go to step 3 and this process will continue until the condition of step 6 is met.

Figure 2 can be used to illustrate the final state estimation algorithm. The steps of this algorithm are the same as the steps of the location algorithm, except that step 8 is expressed as follows: If the main objective function criterion is met, the best location of the devices is determined and the program is completed, otherwise, it will go to step 3 and this process will continue until the main objective function is met.

\section{Simulation and results}

The software verification was performed with many IEEE test networks and a real network in the Yazd Power Distribution Company in [28]. The following is an example implementation of the proposed algorithm for the IEEE 14 Bus network shown in Figure 3. Network information is available in [28] and [29]. To obtain the amplitude and phase values of the bus voltages for the starting point (considering bus 1 as the slag bus), this network is simulated in MATPOWER software. The load distribution results are presented in Table 1. 


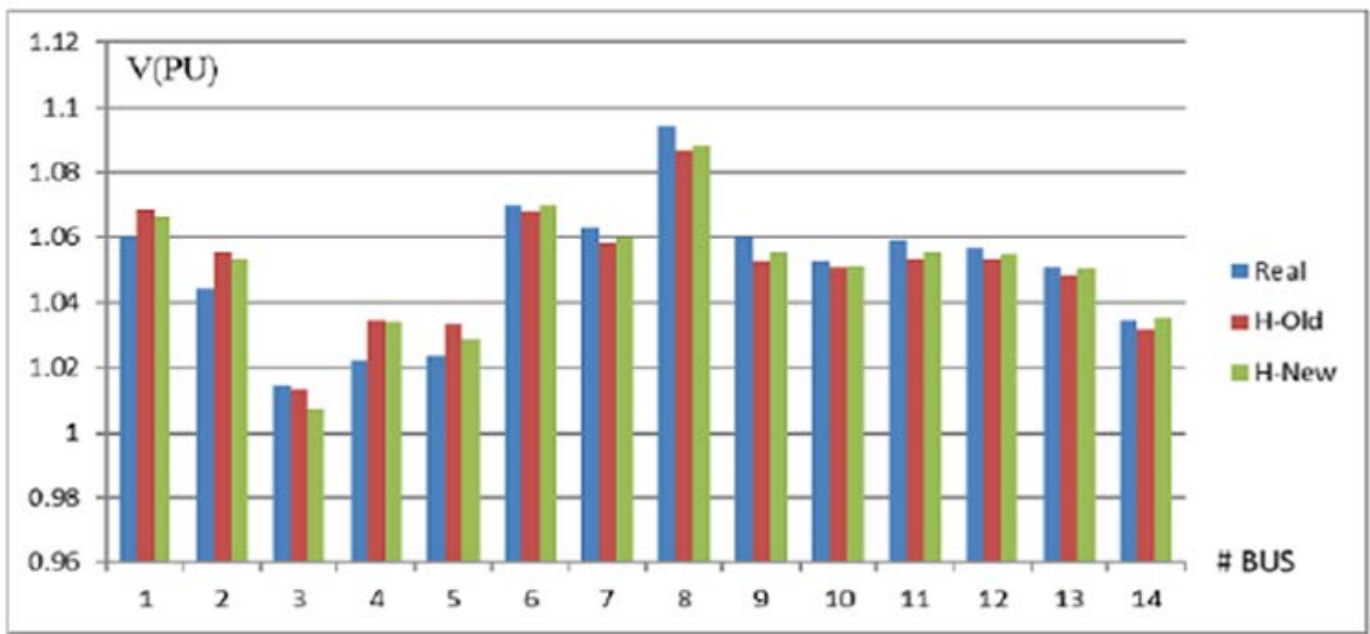

Figure 4. Voltage amplitude difference in 14-bus network by two simulation methods

Table 1. 14-bus network starting point values

\begin{tabular}{|c|c|c|c|c|c|}
\hline $\mathbf{I}$ & $\boldsymbol{V}_{\boldsymbol{i}}(\boldsymbol{p u})$ & $\boldsymbol{\theta}_{\boldsymbol{i}}(\mathbf{D e g})$ & $\mathbf{I}$ & $\boldsymbol{V}_{\boldsymbol{i}}(\boldsymbol{p u})$ & $\boldsymbol{\theta}_{\boldsymbol{i}}(\mathbf{D e g})$ \\
\hline 1 & 1.060 & 0.000 & 8 & 1.090 & -13.360 \\
\hline 2 & 1.045 & -4.983 & 9 & 1.065 & -14.939 \\
\hline 3 & 1.010 & -12.725 & 10 & 1.051 & -15.939 \\
\hline 4 & 1.018 & -10.313 & 11 & 1.057 & -14.791 \\
\hline 5 & 1.020 & -8.784 & 12 & 1.055 & -15.076 \\
\hline 6 & 1.070 & -14.221 & 13 & 1.050 & -15.156 \\
\hline 7 & 1.062 & -13.360 & 14 & 1.036 & -16.036 \\
\hline
\end{tabular}

In this network, the required location and parameters are measured and the state values are determined using the final algorithm. An example of the output of this algorithm is assumed to be PMU in Table 2. According to the information in Table 2 and the fact that the current measurement units should be located in buses 4, 5, 7, and 9, this algorithm specifies that the PMU phasor measurement units in buses 4, 5, 7, and 9 are placed. Simultaneously with determining the number and location of the meters, the state variables are also calculated using the final algorithm. For this purpose, once the calculations have been done using the $\mathrm{H}$ matrix mentioned in (5) and once the calculations have been done using the $\mathrm{H}$ matrix mentioned in (11). It is worth mentioning that because two PMUs are located on both sides of the line, (11) can be used. The results of the algorithm implementation in two modes and the actual results [29] are listed in Table 3. By comparing the results of these two estimation methods with the actual values of the state variables, we can point to the proper performance of the new method (Equation (13)) in improving the load estimation accuracy in power networks. As shown in Table 4, the mean squares of the average size fault of the voltages and their angles in the usual method are $0.58 \%$ and $5.92 \%$, respectively, and in the method presented by this paper $0.45 \%$ and $4.58 \%$. And this indicates an improvement in the accuracy of the results in the proposed method. To illustrate the performance of these two methods, we can refer to the two diagrams in Figures 4 and 5. Figures 4 and 5 show the values of voltages and their angles for all three 
cases in Table 3, in which blue indicates the actual values of the voltage range and phase angle per bus, and red indicates the results of the method. Before or in other words, the H matrix (5), and green indicates the results of using the new method or in other words, the new $\mathrm{H}$ matrix (11). For example, if we look at bus number 5, it is clear that in the innovative method, the estimated value is closer to the actual value than the estimated value of the previous method.

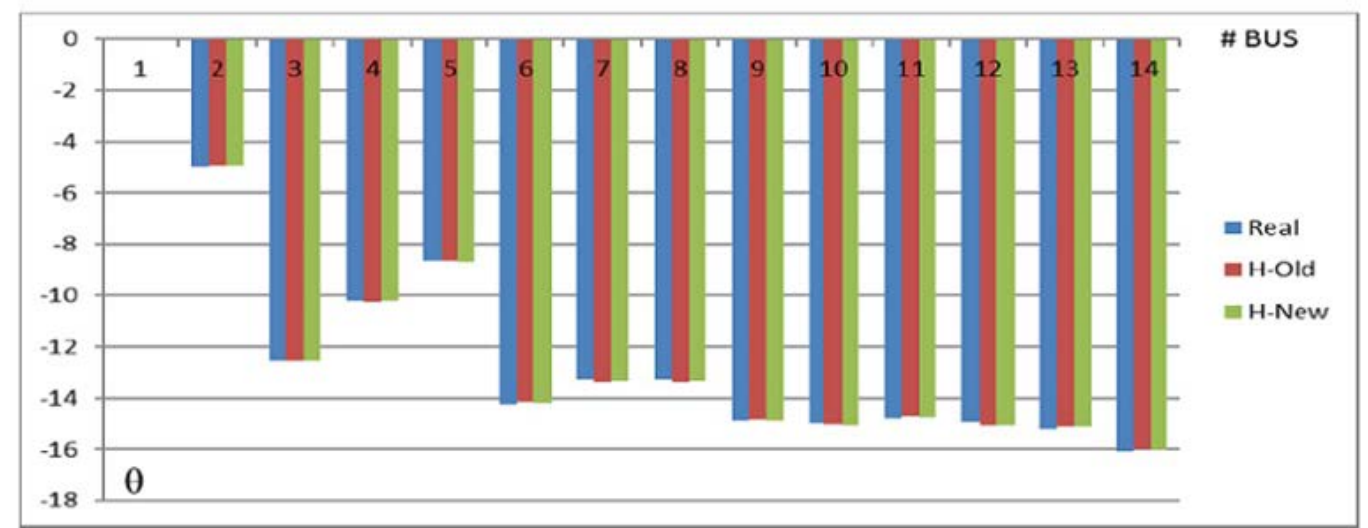

Figure 5. The difference in the phase angle of the buses in the 14-bus network by two simulation methods

Table 2. Number and location of 14-bus network measurement variables with PMU

\begin{tabular}{|c|c|c|c|}
\hline Measurement, i & Type & Measurement, i & Type \\
\hline 1 & $P_{10}$ & 17 & $Q_{4-5}$ \\
\hline 2 & $P_{1-2}$ & 18 & $Q_{4-7}$ \\
\hline 3 & $P_{2-3}$ & 19 & $Q_{4-9}$ \\
\hline 4 & $P_{3-4}$ & 20 & $Q_{6-5}$ \\
\hline 5 & $P_{4-5}$ & 21 & $Q_{6-12}$ \\
\hline 6 & $P_{4-7}$ & 22 & $Q_{7-4}$ \\
\hline 7 & $P_{4-9}$ & 23 & $I_{4-5}$ \\
\hline 8 & $P_{6-5}$ & 24 & $I_{4-7}$ \\
\hline 9 & $P_{6-12}$ & 25 & $I_{5-1}$ \\
\hline 10 & $P_{7-4}$ & 26 & $I_{7-4}$ \\
\hline 11 & $Q_{6}$ & 27 & $I_{7-9}$ \\
\hline 12 & $Q_{9}$ & 28 & $I_{9-4}$ \\
\hline 13 & $Q_{14}$ & 29 & $V_{2}$ \\
\hline 14 & $Q_{1-2}$ & 30 & $V_{3}$ \\
\hline 15 & $Q_{2-3}$ & 31 & $\theta_{4-9}$ \\
\hline 16 & $Q_{3-4}$ & 32 & $\theta_{7-4}$ \\
\hline
\end{tabular}


By comparing the results of the estimated values of the voltage range and phase of the bus with their actual values and considering the two diagrams mentioned, it can be concluded that the new method uses the phase difference equations in the $\mathrm{H}$ matrix to increase the estimation accuracy. The state of a power grid has been very successful. The results of the implementation of the proposed algorithm for the real network presented in [28], similar to the presented results, show an increase in the accuracy of the estimated values.

\section{Conclusion}

Evaluation of state variables of a network is required to control a power system and in control centers, this evaluation is done using state estimation. The state estimate provides the best estimate of the system state vectors (amplitude and phase voltage phase of the buses) according to the measured information in the shortest possible time. In this paper, a new method is presented to obtain a more accurate estimate of all variables in the state of the power grid. In the proposed method, after determining the number and location of measuring devices to make the network visible, the innovative method of adding the derivative of the angle voltage difference of the buses to the $\mathrm{H}$ matrix has been used. Mode estimation was performed by the WLS method and MATPOWER software was used to calculate the starting values as well as the values measured by the measuring devices. The simulated results on this standard network show that the proposed method is successful in increasing the accuracy of the state estimation and reduces the fault of the estimated value of the state variables with its actual value.

Table 3. WLS Estimated Values Actual values of the 14-bus network.

\begin{tabular}{|c|c|c|c|c|c|c|}
\hline \multirow[t]{2}{*}{ I } & \multicolumn{2}{|c|}{ Actual values } & \multicolumn{2}{|c|}{$\begin{array}{l}\text { Estimated values using the old } \\
\qquad \text { H. }\end{array}$} & \multicolumn{2}{|c|}{$\begin{array}{l}\text { Estimated values using the new } \\
\qquad \text { H. }\end{array}$} \\
\hline & $V_{i}(p u)$ & $\overline{\theta_{i}(D e g)}$ & $V_{i}(p u)$ & $\theta_{i}(D e g)$ & $V_{i}(p u)$ & $\overline{\theta_{i}(D e g)}$ \\
\hline 1 & 1.0600 & 0.0000 & 1.0684 & 0.0000 & 1.0664 & 0.0000 \\
\hline 2 & 1.0443 & -4.9645 & 1.0557 & -4.9223 & 1.0537 & -4.9510 \\
\hline 3 & 1.0142 & -12.5525 & 1.0130 & -12.5117 & 1.0070 & -12.5378 \\
\hline 4 & 1.0222 & -10.1950 & 1.0344 & -10.2572 & 1.0343 & -10.2361 \\
\hline 5 & 1.0235 & -8.6384 & 1.0331 & -8.6554 & 1.0289 & -8.6760 \\
\hline 6 & 1.0702 & -14.2147 & 1.0681 & -14.1659 & 1.0699 & -14.1907 \\
\hline 7 & 1.0629 & -12.2643 & 1.0582 & -13.3576 & 1.0598 & -13.3256 \\
\hline 8 & 1.0939 & -12.2874 & 1.0867 & -13.3576 & 1.0883 & -13.3265 \\
\hline 9 & 1.0607 & -14.8839 & 1.0527 & -14.8634 & 1.0554 & -14.9099 \\
\hline 10 & 1.0529 & -14.9728 & 1.0505 & -15.0003 & 1.0511 & -15.0685 \\
\hline 11 & 1.0592 & -14.7978 & 1.0534 & -14.7347 & 1.0557 & -14.7609 \\
\hline 12 & 1.0569 & -14.9195 & 1.0537 & -15.0764 & 1.0551 & -15.0462 \\
\hline 13 & 1.0506 & -15.1871 & 1.0480 & -15.0946 & 1.0503 & -15.1263 \\
\hline 14 & 1.0346 & -16.0643 & 1.0316 & -15.9747 & 1.0353 & -16.00574 \\
\hline
\end{tabular}


Table 4. Mean values of fault estimation of WLS mode variables in 14-bus network

\begin{tabular}{|c|c|c|c|c|}
\hline \multirow[t]{2}{*}{ I } & \multicolumn{2}{|c|}{$\begin{array}{l}\text { Average estimated fault relative } \\
\text { to the actual value using (5) }\end{array}$} & \multicolumn{2}{|c|}{$\begin{array}{l}\text { Average estimated fault relative } \\
\text { to the actual value using (13) }\end{array}$} \\
\hline & $\Delta \mathbf{V}$ & $\Delta \boldsymbol{\theta}$ & $\Delta \mathbf{V}$ & $\Delta \boldsymbol{\theta}$ \\
\hline fault & 0.0058 & 0.0592 & 0.0045 & 0.0458 \\
\hline
\end{tabular}

\section{References}

[1] Golestan, S., Ebrahimzadeh, E., Guerrero, J. M., Vasquez, J. C., Blaabjerg, F., “An adaptive least-error squares filter-based phase-locked loop for synchronization and signal decomposition purposes”, IEEE Transactions on Industrial Electronics, 64(1) (2017) : 336-346.

[2] Xu, S., Liu, H., Bi, T., “A novel frequency estimation method based on complex Bandpass filters for P-class PMUs with short reporting latency”, IEEE Transactions on Power Delivery, doi: 10.1109/TPWRD.2020.3038703.

[3] Sun, Y., et al., "Harmonic contribution evaluation based on the distribution-level PMUs", IEEE Transactions on Power Delivery, doi: 10.1109/TPWRD.2020.2996677.

[4] Peng, L., Zhao, J., Tang, Y., Mili, L., Gu, Z., Zheng, Z., "Real-time LCC-HVDC maximum emergency power capacity estimation based on local PMUs”, IEEE Transactions on Power Systems, doi: 10.1109/TPWRS.2020.3021099.

[5] Risbud, P., Gatsis, N., Taha, A., "Multi-period power system state estimation with PMUs under GPS spoofing attacks”, Journal of Modern Power Systems and Clean Energy, 8(4) (2020) : 597-606.

[6] Lu, Y. et al., "Graph computing based distributed state estimation with PMUs", IEEE Power \& Energy Society General Meeting (PESGM), Montreal, QC, (2020) : 1-5, doi: 10.1109/PESGM41954.2020.9281976.

[7] Wood, A. J., Wollenberg, B. F., "Power generation, operation and control”, 2nd Ed, New York, Wiley, (1996) : 453-513.

[8] Abur, A., Exposito, A. G., "Power system state estimation: Theory and implementation”, New York, Marcel Dekker, (2004).

[9] Georgakopoulos, D., S. Quigg, "Precision Measurement System for the Calibration of Phasor Measurement Units," in IEEE Transactions on Instrumentation and Measurement, 66(6) (2017) : 1441-1445.

[10] Supriya, P., Nambiar, T. N. P., "Harmonic state estimation for a simple power system model using independent component analysis”, Proc. IEEE 9th Int. Conf. on Intelligent Systems and Control, ISCO'15, 4 pp., Coimbatore, India, 9-10 Jan. (2015).

[11] Okon, T., Wilkosz, K., "WLS state estimation in polar and rectangular coordinate systems for power system with UPFC: significance of types of measurements”, Proc. of the Int. Symp. Modern Electric Power Systems, MEPS'10, 6 pp., Wroclaw, Poland, 20-22 Sept. (2010). 
[12] Zhao, J. et al., "Power system dynamic state estimation: Motivations, definitions, methodologies, and future work”, IEEE Transactions on Power Systems, 34(4) (2019) : 3188-3198.

[13] Hajian, M., Ranjbar, A. M., Amaree, T., Mozafari, M., “Optimal placement of PMUs to maintain network observability using a modified BPSO algorithm”, International J. of Electrical Power \& Energy Systems, 33(1) (2011) : 28-34.

[14] Risso, M, Rubiales, A. J., Andres Lotito, P., "Hybrid method for power system state estimation”, Generation, Transmission \& Distribution, IET, 9(7) (2015) : 636-643.

[15] Hurtgen, M., Maun, J. C., “Optimal PMU placement using iterated local search", International J. of Electrical Power \& Energy Systems, 32(8) (2010) : 857-860.

[16] Deoliveira-Dejesus, P., Rodriguez, N., Celeita, D., Ramos, G., "PMU-based system state estimation for multigrounded distribution systems”, IEEE Transactions on Power Systems, doi: 10.1109/TPWRS.2020.3017543.

[17] Qi, J., Sun, K., Kang, W., “Optimal PMU placement for power system dynamic state estimation by using empirical observability gramian”, IEEE Trans. on Power Systems, 30(4) (2015) : 2041- 2054.

[18] Ramesh, L., Choudhury, S. P., Chowdhury, S., Crossley, P. A., "Elecrical power system state estimation meter placement a comparative survey report”, Electric Power Components and Systems, 36(10) (2008) : 1115-1129.

[19] Celik, M. K., Liu, W. -H. E., “An incremental measurement placement algorithm for sate estimation”, IEEE Trans. on Power Systems, 10(3) (1995) : 1698-1703.

[20] Zhang, D., Deng, Z., Wang, B., Fu, M., “The application of square-root cubature Kalman filter in the SINS/CNS integrated navigation system”, Proc. Chinese Guidance Navigation and Control Conference (CGNCC), (2016) : 2331-2335.

[21] Huang, G. M., Jiansheng, L., Abur, A., "A heuristic approach for power system measurement placement design”, Proc. of the 2003 Int. Symp. on Circuits and Systems, ISCAS'03, Bangkok, Thailand, 25-28 May 2003, vol. 3, (2003) : 407-410,

[22] Rakpenthai, C., Premrudeepreechacharn, S., Uatrongjit, S., Watson, N. R., "Measurement placement for power system state estimation by decomposition technique”, Proc. 11th Int. Conf. on Harmonics and Quality of Power, pp. 414-418, Lake Placid, NY, USA, 12-15 Sept. 2004.

[23] Zhao, H., Li, Y., Mi, Z. Yu, L., "Sensitivity constrained PMU placement for complete observability of power systems”, Proc. Transmission and Distribution Conf. and Exhibition: Asia and Pacific, IEEE/PES'05, 5 pp., Dalian, China, 18-18 Aug. 2005.

[24] Madtharad, C., Premrudeepreechacharn, S., Watson, N. R., Ratchai, S. U., “An optimal measurement placement method for power system harmonic state estimation”, IEEE Trans. on Power Delivery, 20(2) (2005) : 1514-1521.

[25] Kumar, A., Das, B., Sharma, J., "Genetic algorithm-based meter placement for static estimation of harmonic sources”, IEEE Trans. on Power Delivery, 20(2) (2005) 10881096.

[26] El-Zonkoly, A., "Optimal meter placement using genetic algorithm to maintain network observability”, Expert Systems with Applications, 31(1) (2006) 193-198.

[27] Li, S., Li, Z., Li, J., Wang, Q., Song, Z., Chen, Z., et al., "Event-based cubature Kalman filter for smart grid subject to communication constraint”, IFAC, 50 (2017) : 49-54. 
[28] Kooshkbaghi, M., Marquez, H. J., "Event-triggered state estimation of high dimensional nonlinear systems with highly nonlinear state space model using cubature Kalman filter”, 2019 IEEE Canadian Conference of Electrical and Computer Engineering (CCECE), Edmonton, AB, Canada, (2019) : 1-4

[29] Gomathi, V., Venkateshkumar, C., Ramachandran, V., "Power systems state estimation with interline power flow controller”, International J. on Electrical \& Power Engineering, 1(2) (2010) : 56-69. 